Review Article

\title{
Breaking the mold: a brief review on the diagnostic and treatment approaches of mucormycosis
}

\author{
Aroop Mohanty ${ }^{1 *}$, Pratima Gupta ${ }^{2}$, Saurabh Varshney $^{3}$, Ankita Kabi ${ }^{4}$, Sumeet Angral ${ }^{5}$
}

\author{
${ }^{1}$ Department of Microbiology, AIIMS, Gorakhpur, Uttar Pradesh, India \\ ${ }^{2}$ Department of Microbiology, AIIMS, Rishikesh, Uttarakhand, India \\ ${ }^{3}$ Department of ENT, AIIMS Deoghar, Jharkhand, India \\ ${ }^{4}$ Department of Emergency Medicine, AIIMS, Rishikesh, Uttarakhand, India \\ ${ }^{5}$ Department of ENT, AIIMS, Deoghar, Jharkhand, India
}

Received: 27 May 2021

Revised: 02 June 2021

Accepted: 15 April 2021

\author{
*Correspondence: \\ Dr. Aroop Mohanty, \\ E-mail: aroopmohanty7785@yahoo.com
}

Copyright: () the author(s), publisher and licensee Medip Academy. This is an open-access article distributed under the terms of the Creative Commons Attribution Non-Commercial License, which permits unrestricted non-commercial use, distribution, and reproduction in any medium, provided the original work is properly cited.

\begin{abstract}
Mucormycosis is a severe emerging angioinvasive fungal infection that occurs predominantly in immunocompromised individuals. The incidence of this disease seems to be increasing in recent years with the emergence of new causative agents and rise of the susceptible population. The present COVID-19 pandemic has seen an un-precented increase in the number of mucormycosis cases all over the world. It has emerged as one of the most common complication of COVID-19 leading to severe morality. Uncontrolled diabetes has been postulated to be the most common underlying disease in developing countries and hematological malignancies in case of the high-income countries. Besides diabetes, chronic renal failure and tuberculosis have also been predisposed in the Indian subcontinent. Rhino-cerebral form is mostly seen in diabetics whereas the pulmonary type is the most common presentation in patients with hematological malignancies and solid organ transplant. Diagnosis and treatment of this rapidly progressive disease is equally challenging. Microscopy and culture are the cornerstone for the diagnosis, but if assisted by imaging and molecular assays, it plays an important role in the correct and early identification of the causative agent. Successful management of mucormycosis is based on an integrated approach, including reversal or discontinuation of underlying risk factors, early administration of antifungal agents, and complete removal of infected tissue and use of various adjunctive therapies.
\end{abstract}

Keywords: Mucormycosis, Aseptate hyphae, Mucoraeles, Diabetes mellitus

\section{INTRODUCTION}

Mucormycosis is a rare, emerging, rapidly progressive and destructive infective disease with high morbidity and mortality caused by fungi belonging to the order Mucorales, subphylum Mucoromycotina. It is increasingly reported in patients with diabetes mellitus, immunecompromised state, hematological malignancy, solid organ transplants and corticosteroid therapy. ${ }^{1}$ Based on the anatomical site of involvement, it is classified into various types. The most common presentation of them is rhino- orbital-cerebral type followed by others. Initially, the term 'mucormycosis' was used for a long time and was then supplanted by the term 'zygomycosis', and currently, based on molecular studies, 'mucormycosis' is the appropriate term. ${ }^{2}$

Upon suspicion of mucormycosis in a patient it is strongly recommended that proper imaging be carried out to document extent of disease. Since delay in the start of treatment is associated with increased mortality, a rapid diagnosis followed by therapeutic intervention including 
the immediate involvement of a multidisciplinary approach would result in better survival. This review will outline the various aspects of mucormycosis including epidemiology, pathogenesis, clinical features as well as the areas of research targeting diagnosis and the new modalities of treatment for this frequently lethal disease.

\section{METHODS}

In this review, a comprehensive literature search was done in order to analyze and better understand the epidemiology, clinical manifestations, laboratory diagnosis and treatment approaches of mucormycosis. Five independent researchers searched through the literature, three of them collected all relevant articles and the rest two reviewed all the selected abstracts. Literature for this review was identified by searching the following online databases: pubmed, google scholar, scopus and embase. We searched the scientific publications using the MESH terms rhizopus, zygomycetes, zygomycosis, mucorales and mucormycosis, amphotericin-B. Based on the main research objectives, articles were classified into one of the following research domains: epidemiology, clinical manifestations, diagnosis, treatment or prevention and control. 'Epidemiology' included studies on the epidemic distribution (when, where, who); clinical manifestations and diagnosis included studies on the clinical features (signs and symptoms) and laboratory diagnosis; and prevention control included studies on prevention, control and treatment measures.

\section{MYCOLOGY}

The fungi belonging to mucorales cause most human infections predominantly by inhalation of sporangiospores that have been released in air and from traumatic inoculation of organism into disrupted skin or ingestion of contaminated food. ${ }^{3}$ These are ubiquitous in nature and can be found on soil and decaying vegetation. They grow rapidly and release large numbers of spores, which can become airborne; hence all humans have ample exposure to these fungi during day-to-day activities. Around 11 genera and 27 species have been associated with human beings of which Rhizopus arrhizus is the commonest globally. Other species like rhizomucor, mucor, Lichtheimia, Apophysomces and Cunninghamella are also important etiological agents. ${ }^{4,5}$

This varies according to the region; for example, Rhizopus spp, Mucor spp and Lictheimia spp are commonly identified in Europe whereas Rhizopus spp followed by Apophysomyces spp and Mucor spp are more prevalent in Indian subcontinent.The hyphae of the mucorales are distinct and help in presumptive identification from clinical specimens. They are broad (5 to 15-micron diameter) and appear as aseptate or sparsely septate ribbon like hyphae. This is in contrast with the hyphae of Aspergillus, which are narrower (2-to-5-micron diameter), exhibit regular branching, and have many septations. The lack of regular septations may contribute to the fragile nature of the hyphae and the difficulty of growing them from clinical specimens. Grinding clinical specimens can cause excessive damage to the hyphae thus finely mincing tissues is preferred for culturing tissue samples that may contain molds.

\section{EPIDEMIOLOGY}

The incidence is rising globally but there has been an alarming upsurge of cases in India and China among patients with uncontrolled diabetes mellitus. ${ }^{6-8}$ However, on the contrary a recent study illustrates that the disease burden is higher in Europe (34\%) than in Asia (31\%) and North or South America (28\%). ${ }^{9}$ There is a contrasting difference in the underlying risk factors between the Asian region and the Western world. Diabetes mellitus continues to be the single most important risk factor in our region, contrasting to hematological malignancy and transplant recipients in USA and Europe. ${ }^{10,11}$ A considerable number of cases are also seen in the immunocompetent population without any known underlying illness. Trauma has been postulated as the crucial predisposing factor in such patients who present with cutaneous mucormycosis. In recent times, nosocomial mucormycosis has also been on the rise. In recent years, health-care-associated mucormycosis is also on the rise. ${ }^{12}$

These are those cases which are associated with healthcare procedure (medical devices and surgery, including diagnostic or therapeutic invasive procedures) with an identified or suspected source of infection. ${ }^{13,14}$ Diabetes mellitus and solid organ transplant are again two of the most critical risk factors noted in such group of patients. The major underlying diseases/risk factors associated with mucormycosis in different countries are illustrated in Table 1 .

Table 1: Underlying disease/risk factors for mucormycosis in the world.

\begin{tabular}{|c|c|}
\hline Country & Underlying disease/ risk factor \\
\hline \multirow{2}{*}{ India $^{15}$} & Diabetes mellitus \\
\hline & Chronic kidney disease \\
\hline Iran $^{16}$ & Diabetes mellitus \\
\hline \multirow{2}{*}{ Australia $^{17}$} & Corticosteroid therapy \\
\hline & Neutropenia \\
\hline \multirow{2}{*}{ Turkey $^{18}$} & Hematological malignancy \\
\hline & Neutropenia \\
\hline \multirow{2}{*}{$\mathbf{U S A}^{19}$} & Diabetes mellitus \\
\hline & Hematological malignancy \\
\hline \multirow{2}{*}{ Egypt $^{20}$} & Solid organ transplantation \\
\hline & Corticosteroid therapy \\
\hline \multirow{2}{*}{ Greece $^{21}$} & Immunocompetent host \\
\hline & Hematological malignancy \\
\hline \multirow{2}{*}{ Italy ${ }^{22}$} & Hematological malignancy \\
\hline & Corticosteroid therapy \\
\hline \multirow{2}{*}{ France $^{23}$} & Neutropenia \\
\hline & Hematological malignancy \\
\hline
\end{tabular}




\section{PATHOGENESIS}

Rhizopus organisms have an enzyme, ketone reductase, which allows them to thrive in high glucose, acidic conditions. Serum from healthy individuals inhibits the growth whereas serum from individuals in diabetic ketoacidosis stimulates growth of rhizopus. ${ }^{24}$ Rhinoorbital-cerebral and pulmonary mucormycosis are acquired by the inhalation of spores. In healthy individuals, cilia transport these spores to the pharynx, and they are cleared through the gastrointestinal tract. In susceptible individuals, infection usually begins in the nasal turbinates or the alveoli. ${ }^{25}$ The agents of mucormycosis are angioinvasive; thus, infarction of infected tissues is the hallmark of invasive disease. ${ }^{26}$

Iron overload and deferoxamine therapy play as major part in the pathogenesis of mucormycosis. Patients with uncontrolled diabetes, acidotic conditions, renal failure and transfusion related disorders who are on or treated with deferoxamine promote aggressive invasive growth of acquired Mucorales spores. The excessive glycosylation of proteins such as transferrin and ferritin, owing to poorly controlled diabetes, results in decreased affinity of these proteins to bind iron, making that important element available for mucorales. Lower blood $\mathrm{pH}$ due to ketoacidosis and other forms of acidosis compromises the affinity of transferrin to bind iron. Finally, the iron removed by deferoxamine is captured by siderophores on rhizopus species and this iron helps in the growth of these fungi.

\section{CLINICAL MANIFESTATIONS}

Mucormycosis is classified into rhino-orbito-cerebral (ROCM), pulmonary, gastrointestinal, cutaneous, renal, disseminated and other miscellaneous forms which include infection of bones, heart, parotid gland, uterus and lymph nodes. ROCM is the most common form and it is often seen in patients with diabetic ketoacidosis or with uncontrolled diabetes mellitus

Prolonged fever is seen in most patients, although some patients might be asymptomatic. This typically develops in patients with profound neutropenia and graft-versus-host disease, whereas diabetic patients usually present with rhino-orbital disease. ${ }^{27}$ Pulmonary mucormycosis can spread contiguously into other organs, for example, through the diaphragm into the abdomen or to the mediastinum and the heart. ${ }^{28,29}$ Most of these patients present with fever and massive hemoptysis. ${ }^{30}$ The most common risk factors associated are hematological malignancies, solid organ transplantations and treatment with glucocorticoids. ${ }^{31,32}$

Cutaneous and soft-tissue mucormycosis are the most common forms of mucormycosis in immunocompetent patients, primarily after skin disruption due to traumatic injury (e.g., from natural disasters, motor vehicle accidents, improvised explosive devices in theatres of war, or iatrogenic sources), surgery, or burns. Abscesses, skin swelling, necrosis, dry ulcers, and eschars are characteristic presentations. ${ }^{33-36}$ Patients of Gastrointestinal (GI) mucormycosis generally present with abdominal pain, gastrointestinal bleed, abdominal distension and diarrhea. It is commonly seen in low birth infants and in patients undergoing peritoneal dialysis. Renal mucormycosis lately has seen a rise in incidence, but is not associated with any underlying illness. Fever, flank pain and anuria are the common presenting symptoms. Due to the angioinvasive nature of these fungi, disseminated mucormycosis have also been reported with lungs being the most common site followed by central nervous system (CNS), liver and kidney.

Survival rates in mucormycosis remains poor and overall mortality rates for mucormycosis range from $40 \%$ to $80 \%$ with varying rates depending on underlying conditions and sites of infection. The highest survival rates are reported in patients with a healthy immune status and those without comorbidities. The worst prognosis is observed in patients with hematological malignancies and HSCT recipients and extensive burns. Disseminated disease, especially of CNS, is associated with mortality rates higher than $80 \%$. Generally, improved survival is related to earlier diagnosis and application of early, multidisciplinary treatment approaches involving aggressive surgical debridement.

An emerging species, Mucor irregularis causes an infection completely different from epidemiology and clinical presentation. Unlike the others which are rapidly progressive, it is chronic in nature and persists for years in immunocompetent patients without any apparent risk factors. It affects the skin and subcutaneous tissue ultimately leading to sever disfigurement. ${ }^{37}$

Here we discuss two clinical cases of mucormycosis:

\section{Case 1}

49-years female with uncontrolled type 2 diabetes mellitus, presented with 5 days history of bilateral loss of vision and drooping of right eyelid, 4 days history of left eye swelling and headache associated with fever and vomiting. On examination there was left eye chemosis, discoloration over left side of face, absent extraocular movements, bilateral pupils fixed and not reacting to light. (Figure $1 \mathrm{~A}-\mathrm{B}$ )

On diagnostic nasal endoscopy, a blackish discoloration was seen over bilateral septal mucosa, left inferior and middle turbinates (Figure 1C). Swab was sent for $\mathrm{KOH}$ which showed broad aseptate hyaline fungal hyphae. On confirmation of diagnosis, left suuprastructural maxillectomy with left orbital exenteration with excision of septum and left cheek was done (Figure 1D). Medical management in the form of liposomal amphotericin and insulin with daily RBS and renal function monitoring was done. 


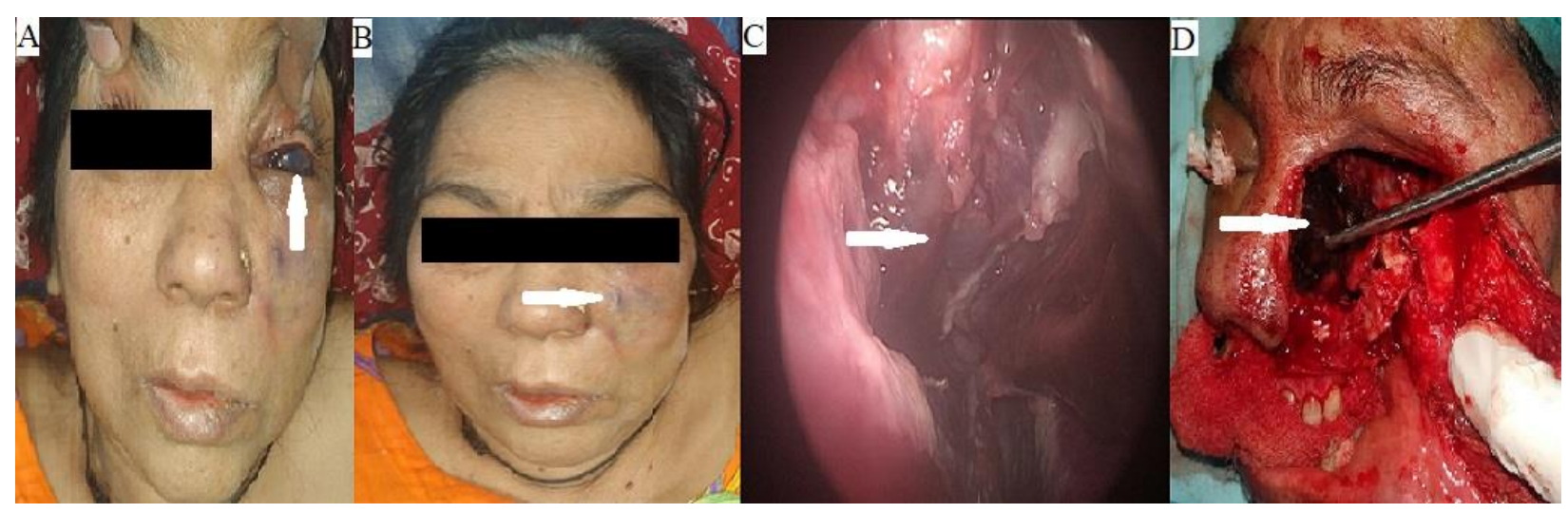

Figure 1: (A, B) Clinical images of patient showing left sided facial discoloration and left eye chemosis; (C) DNE image with blackish eschar over septal mucosa and middle turbinate; (D) Intraoperative image with debridement of diseased tissue (white arrow).

\section{Case 2}

56-years-old male patient, with 20 days history of right sided facial pain with numbness and 7 days history of patch on hard palate. Patient had history of tobacco chewing for last 20 years. On examination a deep, partially black patch was seen over the hard palate (Figure $2 \mathrm{~A}$ and B). Rest of the ENT examination (endoscopic examination), ophthalmologic examination, hematological investigations, X-ray chest and CT Nose and PNS were with in normal limits. Biopsy of the palatine lesion was taken in view of its being neoplastic lesion of the hard palate. On HPE large broad non-septate hyphae invading the blood vessels were seen. Fungal culture on Sabouraud's 'dextrose agar medium' revealed it as Rhizopus microsporum and diagnosis of mucormycosis was established. Patient was treated with surgical debridement twice, followed by Amphotericin B 1 $\mathrm{mg} / \mathrm{kg} /$ day for 6 weeks. Patient was followed up for 4 months with no signs of recurrence and was subsequently fitted with palatal obturator. ${ }^{38}$

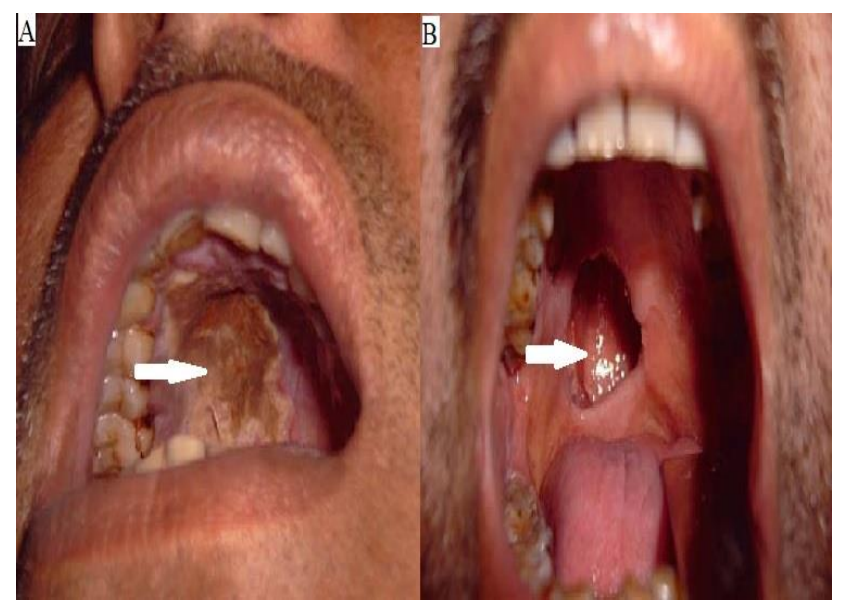

Figure 2: (A) Clinical image if patient showing eschar over hard palate; (B) Oroantral fistula after debridement of the necrosed tissue over hard palate (white arrow).

\section{MUCORMYCOSIS IN INDIA}

An upsurge has been seen all over the world over the past two decades, however the rise in developing countries including India has been alarming with uncontrolled diabetes being the most critical risk factor. ${ }^{39}$ In fact, India has the second largest diabetic population globally with about $70 \%$ of these cases being of uncontrolled diabetes. ${ }^{40}$ The prevalence of mucormycosis is about 140 cases per million population, with the prevalence ranging between $1,37,807$ cases to $2,08,177$ per year. ${ }^{41}$ The rhino-orbitalcerebral form remains the most common presentation. Isolated renal mucormycosis amongst immunocompetent, young individuals is also an emerging entity. The disease is highly aggressive, but the mode of acquisition and spread of the fungus through the body are not yet known. In contrast to other countries, cutaneous infections in apparently healthy individuals due to traumatic implantation of Apophysomyces elegans are a common finding in India.

\section{DIAGNOSIS}

The pre-requisites for the diagnosis of mucormycosis are a high index of suspicion, recognition of host factors, and prompt assessment of clinical manifestations. It depends on the availability of imaging techniques, trained personnel and mycological and histopathological investigations. Microscopy examination and culture

Microscopy includes direct examination and histopathology of the clinical samples received. Along with culture these two are the cornerstones of diagnosing mucormycosis. Direct microscopy of samples using fluorescent brighteners such as Blankophor and Calcofluor White gives us a presumptive diagnosis. The hyphae are seen as non-septate or pauci-septate and having variable width of about 6-25 $\mu \mathrm{m}$ arranged in a ribbon-like structure. These are mostly bifurcated at right angles (Figure 3). Commercial methods such as homogenization of tissue should be avoided before culturing as the hyphae are friable in nature and may be damaged during 
manipulation. For culture, they are routinely inoculated on Sabouraud's dextrose agar and incubated at $30^{\circ} \mathrm{C}$ and $37^{\circ} \mathrm{C}$ separately (Figure 4). The growth is rapid (3 to 7 days) and once it is obtained, LPCB mounts are performed to identify the etiological agent (Figure 5-7).

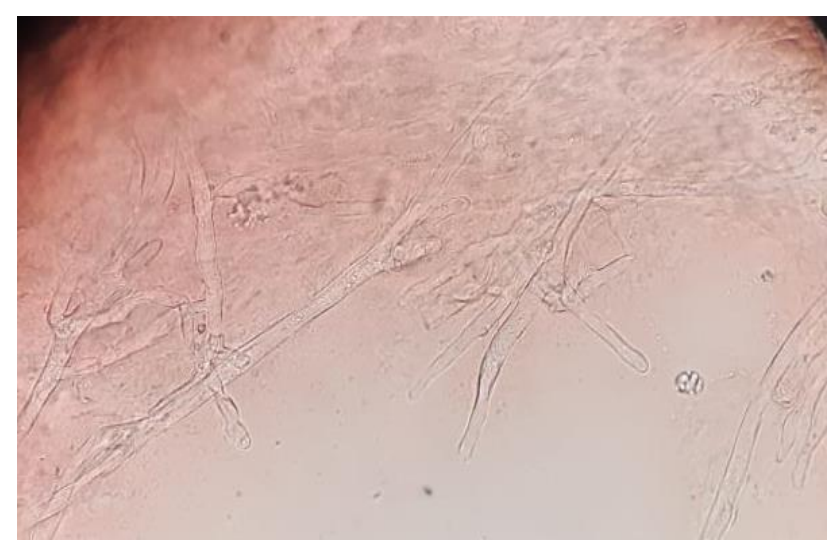

Figure 3: KOH mount showing broad, aseptate acute angled branched fungal hyphae.

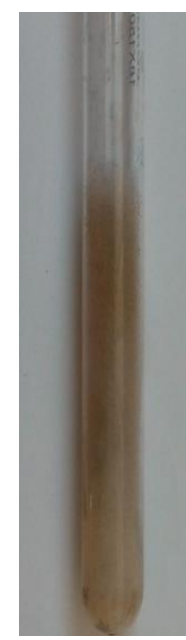

Figure 4: SDA showing white cottony growth of Mucor spp.

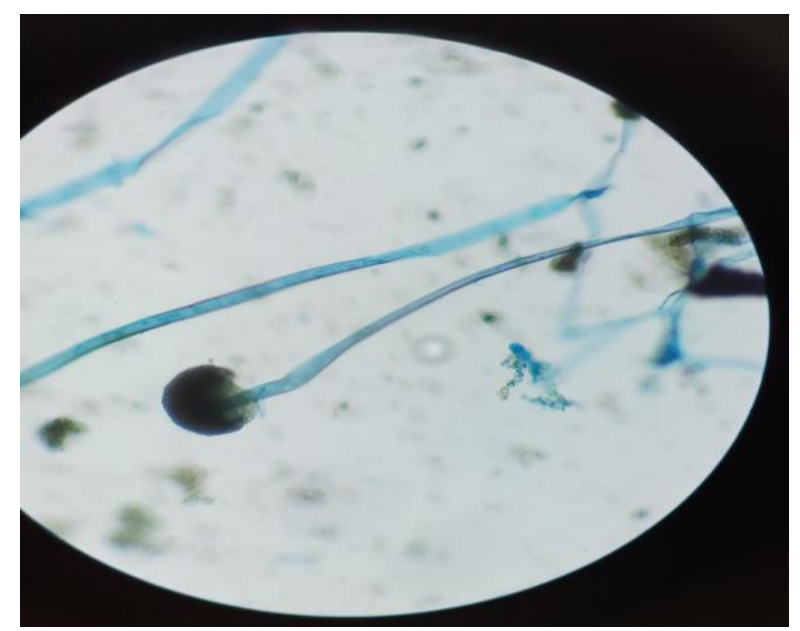

Figure 5: LPCB mount of mucor.

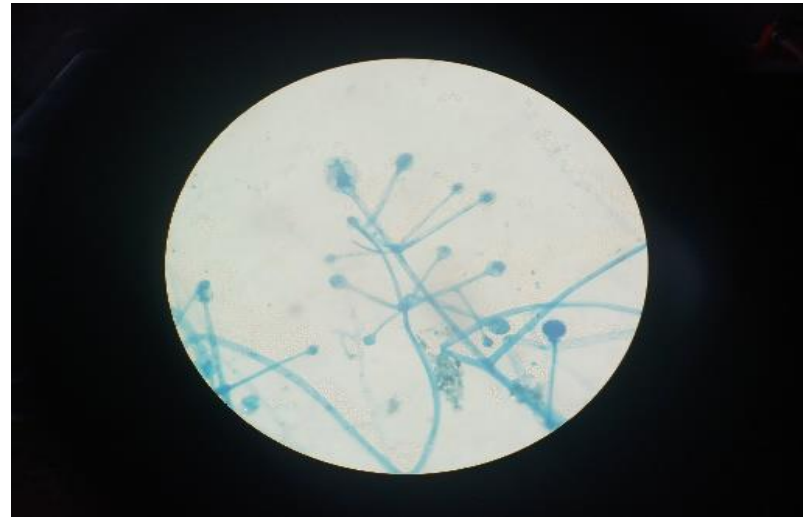

Figure 6: LPCB mount of Lichtheimia corymbiferans.

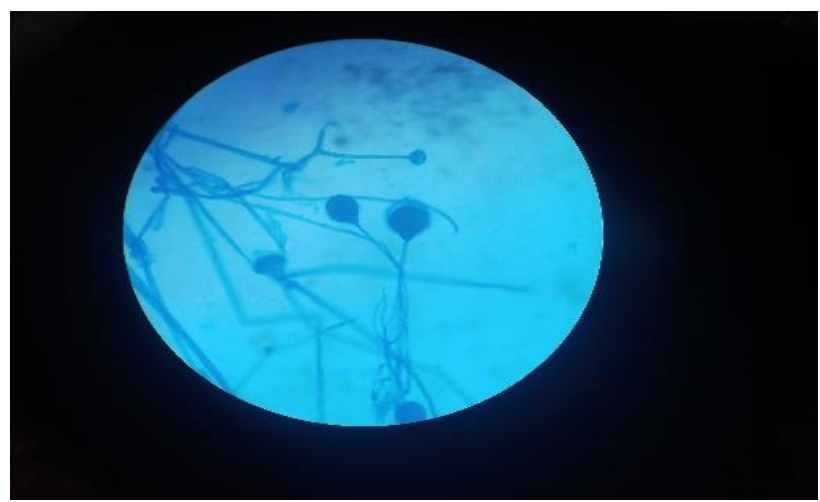

Figure 7: LPCB mount of Apophysomyces elegans.

\section{Histopathology}

Obtaining a diagnosis of mucormycosis on a histomorphological basis is challenging. However, it is crucial in order to prove the fungus as a pathogen from a culture contaminant. To confirm an infection, non-pigmented hyphae showing tissue invasion must be demonstrated in tissue sections stained with hematoxylin-eosin (HE), periodic acid-Schiff stain (PAS), or Grocott-Gomori's methenamine-silver stain (GMS), or both. ${ }^{42}$ In tissue, the hyphae appear ribbon-like with an irregular pattern of branching (Figure 8).

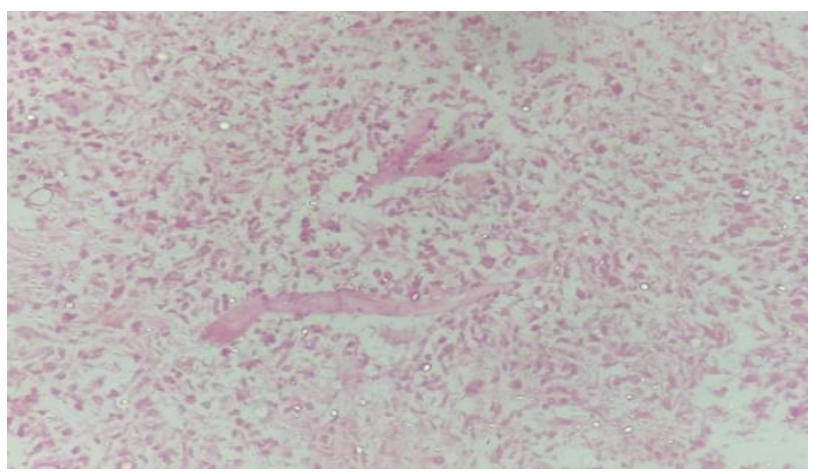

Figure 8: Broad aseptate hyphae seen on $H$ and E stain. 
Sometimes they may appear to have septations as tissue can fold over itself during processing, which may create artificial lines that can be confused with septations. The historically described $90^{\circ}$ branching angle of mucorales in tissue, versus $45^{\circ}$ branching angle of septate molds, can be challenging to identify in tissue due to interstitial pressures exerted on the fungi and alterations in tissue architecture during processing. Thus, the broader and irregular (ribbonlike) nature of the hyphae are more reliable distinguishing characteristics than septations and angle of branching.

In acute lesions, hemorrhagic infarction, coagulation necrosis, angioinvasion, infiltration by neutrophils, and perineural invasion are characteristic features; whereas, in chronic lesions, a pyogranulomatous inflammation with presence of giant cells, and sometimes hyphae covered by the Splendore-Hoeppli phenomenon, which describes deeply eosinophilic material surrounding the pathogen, are seen. ${ }^{43-45}$ It is indeed challenging to diagnose mucormycosis on histo-morphological basis and the most common cause for incorrect morphological diagnosis is misidentification of mucorales as Aspergillus Spp. It is strongly recommended to confirm the diagnosis of mucormycosis in tissue by culture or by application of molecular or in-situ identification techniques, at centers where such assays are available.

\section{Imaging}

Cranial CT or MRI is strongly recommended to determine if sinusitis is present in diabetic patients with facial pain, sinusitis, proptosis, ophthalmoplegia, or newly diagnosed amaurosis. If sinusitis is diagnosed, endoscopy should be done to diagnose mucormycosis. If disease of the eye or brain is suspected, MRI should be conducted instead of a CT scan because of greater sensitivity. If mucormycosis is a potential diagnosis, a biopsy is strongly recommended and once mucormycosis has been proven in a patient with underlying malignancy, cranial, thoracic and abdominal imaging studies to determine the extent of disease are recommended. In patients with hematological malignancy and suspected pulmonary mucormycosis, the HRCT chest is recommended for the detection of the reversed halo sign (RHS), an area of ground-glass opacity surrounded by a ring of consolidation on thoracic CT or vessel occlusion on CT pulmonary angiography. In view of the rapid progression of the disease, weekly CT scans are recommended mostly in unstable patients. ${ }^{46}$ Another emerging imaging technique, which may eventually aid in the diagnosis is the positron emission tomographycomputed tomography (PET/CT) with [18F]fluorodeoxyglucose (FDG).

On CT imaging, the involved areas present as minimally enhancing hypodense soft tissue thickenings (Figure 9) while MRI shows T2 isointense to mildly hypointense soft tissue thickening and heterogeneous post contrast enhancement as the main finding. Bone erosions are seen in $40 \%$ patients, with rest $60 \%$ of the patients showing extrasinus extension across grossly intact appearing bones on imaging. ${ }^{47}$

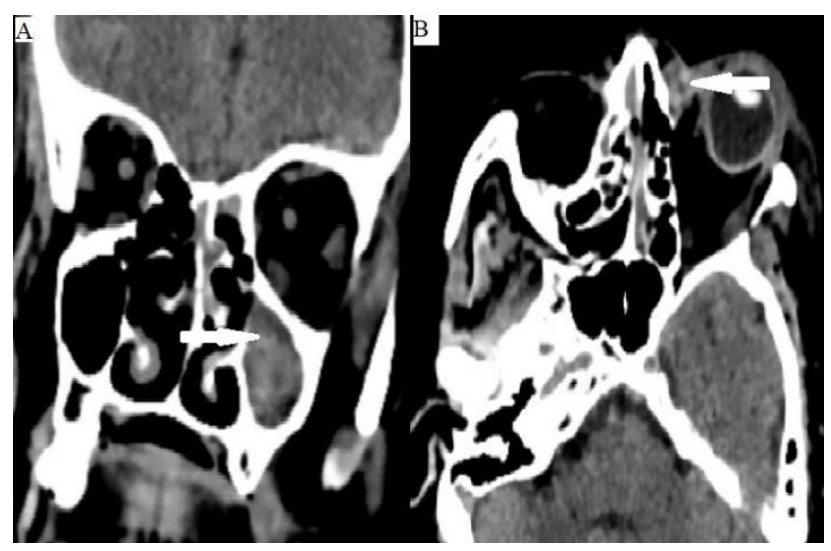

Figure 9: (A) CT nose and PNS showing hypodense soft tissue density in left side maxillary sinus; (B) Left sided periorbital hypodense soft issue thickening (white arrow).

\section{Molecular-based diagnosis}

Currently, in the absence of a standardized test, the use of molecular methods on both fresh clinical material and paraffin sections for the diagnosis of mucormycosis is moderately supported. Fresh material is preferred over paraffin-embedded tissue because formalin damages DNA. Detection of DNA in blood and serum as well as in other body fluids have yielded promising clinical data. Matrix-assisted Laser Desorption/Ionization-Time of Flight mass spectrometry (MALDI-TOF MS) have also revolutionized the early detection of these agents by the use of the extended method of extraction. ${ }^{49}$

\section{Genus and species identification}

Although some genera, such as Cunninghamella, can be associated with an increased mortality rate in patients and are more virulent in experimental models, there is currently sparse evidence that identification of the causative genus or species level, or both, could guide the choice of the antifungal treatment. By contrast, identification to the species level is of importance for improved epidemiological knowledge of the disease. Moreover, species identification is valuable for investigation of healthcare-associated mucormycosis and outbreaks.

Molecular identification is strongly supported and preferred over morphology. The best technique for molecular identification is internal transcribed spacer (ITS) sequencing. MALDI-TOF MS identification is a strong tool bit is not yet validated for all mucorales and mainly relies on in-house databases. In addition, it is an expensive instrument and many laboratories do not have that capacity. ${ }^{49}$ 


\section{TREATMENT APPROACHES}

The treatment of mucormycosis depends on antifungal drugs and surgery. An early complete surgical treatment for mucormycosis whenever possible, in addition to systemic antifungal therapy, is the cornerstone of the treatment of mucormycosis. In any immunocompromised patient with suspected mucormycosis, immediate treatment initiation is strongly recommended. Every attempt to attain a diagnosis should be made at the time of initiation of therapy, but should not delay therapy.

First-line treatment with liposomal amphotericin with daily doses ranging from $1 \mathrm{mg} / \mathrm{kg} /$ day to $10 \mathrm{mg} / \mathrm{kg} /$ day is strongly recommended in all types of organ involvement. ${ }^{50-52}$ If substantial renal toxicity develops, the dose can be reduced as necessary. Amphotericin B lipid complex $5 \mathrm{mg} / \mathrm{kg}$ per day is recommended with moderate strength for patients without CNS involvement. ${ }^{53-54}$ Use of amphotericin B deoxycholate is discouraged because of its toxicity whenever alternatives are available. In kidney transplant recipients, amphotericin B lipid complex 10 $\mathrm{mg} / \mathrm{kg}$ per day has been given. As a salvage treatment, isavuconazole is recommended with moderate strength for the first-line treatment of mucormycosis. In contrast with other mould active azoles, it is less hepatotoxic although it can shorten the QTc interval. Posaconazole oral suspension and delayed-release tablets and infusion has also been marginally supported for use as first-line treatment.

There are no definitive data to guide the use of antifungal combination therapy. Limited data support combinations of polyenes and azoles or polyenes plus echinocandins. Combination therapy can be rationally given due to a lack of enhanced toxicity with possible but unproven benefit; however, data are too limited to support this beyond a marginal recommendation.

The duration of therapy of mucormycosis is unknown and is a personalized decision. The guideline group strongly supports treatment until the permanent reversal of immunosuppression, and there is a complete response on imaging, which might be challenging to determine because of scarring and postoperative changes. There is moderate support for intravenous treatment until stable disease is achieved. In general, weeks to months of therapy are given. If the immune defect is resolved-e.g., diabetes is controlled, neutropenia is definitively resolved, immunosuppression can be tapered or stopped, therapy can be continued until resolution of signs and symptoms of infection, and substantial radiographically improvement. Across several posaconazole oral suspension studies, treatment duration ranged from one week to almost three years; the mean duration was approximately six months. The wide range reflects the pattern of organs involved, with competing risks from underlying conditions. Late relapse in long-term survivors has been documented. When switching to oral treatment, the use of isavuconazole or posaconazole delayed-release tablets is strongly supported. Posaconazole oral suspension can be used but is marginally supported, especially when formulations with higher exposure are available.

\section{PROPHYLAXIS}

In neutropenic patients or those with graft versus host disease, primary prophylaxis with posaconazole oral suspension or delayed-release tablets is recommended to prevent mucormycosis. In immunosuppressed patients with a previous diagnosis of mucormycosis, wide surgical resection and continuation or restart of the last drug effective in that patient is strongly recommended.

\section{CONCLUSION}

Mucormycosis is manifested by a variety of syndromes, particularly in those with diabetes mellitus and in immunocompromised patients. It is characterized by infarction and necrosis of host tissues that result from angio-invasion by hyphae. The most common clinical presentation of mucormycosis is rhino-orbital-cerebral infection. Mucormycosis can also cause pulmonary, gastrointestinal, cutaneous, renal, and disseminated disease. The diagnosis of mucormycosis relies upon the identification of organisms in tissue by histopathology with culture confirmation. However, culture often yields no growth, and histopathologic identification of an organism with a structure typical of Mucorales may provide the only evidence of infection. Treatment of mucormycosis involves a combination of aggressive surgical debridement of involved tissues and antifungal therapy. The drug of choice for initial treatment of is a lipid formulation of amphotericin B. Overall, mortality from rhino-orbital-cerebral mucormycosis ranges from 25 to 62 percent. The prognosis is exceptionally low for patients with brain, cavernous sinus, or carotid involvement, although some patients with these complications have been cured of the infection.

Funding: No funding sources

Conflict of interest: None declared

Ethical approval: Not required

\section{REFERENCES}

1. Prakash H, Ghosh AK, Rudramurthy SM, Singh P, Xess I, Savio J, et al. A prospective multicenter study on mucormycosis in India: Epidemiology, diagnosis, and treatment. Med Mycol. 2019;57(4):395-402.

2. Chung KJ. Taxonomy of fungi causing mucormycosis and entomophthoramycosis (zygomycosis) and nomenclature of the disease: molecular mycologic perspectives. Clin Infect Dis. 2012;54(1):8-15.

3. Richardson M. The ecology of the Zygomycetes and its impact on environmental exposure. Clin Microbiol Infect. 2009;15(5):2-9.

4. Jeong W, Keighley C, Wolfe R, Lee WL, Slavin MA, Kong DCM, et al. The epidemiology and clinical 
manifestations of mucormycosis: a systematic review and meta-analysis of case reports. Clin Microbiol Infect. 2019;25(1):26-34.

5. Prakash H, Chakrabarti A. Global Epidemiology of Mucormycosis. J Fungi. 2019;5(1):26.

6. Saegeman V, Maertens J, Meersseman W, Spriet I, Verbeken E, Lagrou K. Increasing incidence of mucormycosis in University Hospital, Belgium. Emerg Infect Dis. 2010;16(9):1456-8.

7. Guinea J, Escribano P, Vena A, Munoz P, Martínez JMDC, Padilla B, Bouza E. Increasing incidence of mucormycosis in a large Spanish hospital from 2007 to 2015: Epidemiology and microbiological characterization of the isolates. PLoS One. 2017;12(6):179136.

8. Chakrabarti A, Chatterjee SS, Das A, Panda N, Shivaprakash MR, Kaur A, et al. Invasive zygomycosis in India: experience in a tertiary care hospital. Postgrad Med J. 2009;85(1009):573-81.

9. Skiada A, Rigopoulos D, Larios G, Petrikkos G, Katsambas A. Global epidemiology of cutaneous zygomycosis. Clin Dermatol. 2012; 30: 628-632.

10. Chakrabarti A, Das A, Mandal J, Shivaprakash MR, George VK, Tarai B, Rao P, et al. The rising trend of invasive zygomycosis in patients with uncontrolled diabetes mellitus. Med Mycol. 2006;44(4):335-42.

11. Kontoyiannis DP, Yang H, Song J, Kelkar SS, Yang $\mathrm{X}$, Azie N, et al. Prevalence, clinical and economic burden of mucormycosis-related hospitalizations in the United States: a retrospective study. BMC Infect Dis. 2016;16(1):730.

12. Lamaris GA, Ben AR, Lewis RE, Chamilos G, Samonis G, Kontoyiannis DP. Increased virulence of Zygomycetes organisms following exposure to voriconazole: a study involving fly and murine models of zygomycosis. J Infect Dis. 2009;199(9):1399-406.

13. Rammaert B, Lanternier F, Zahar JR, Dannaoui E, Bougnoux ME, Lecuit $M$, et al. Healthcareassociated mucormycosis. Clin Infect Dis. 2012;54(1):44-54.

14. Mohanty A, Kabi A, Mohanty AP. Health problems in healthcare workers: A review. J Family Med Prim Care. 2019;8(8):2568-72.

15. Chakrabarti A, Das A, Sharma A, Panda N, Das S, Gupta KL, Sakhuja V. Ten years' experience in zygomycosis at a tertiary care centre in India. J Infect. 2001;42(4):261-6.

16. Dolatabadi S, Ahmadi B, Rezaei MA, Zarrinfar H, Skiada A, Mirhendi H, et al. Mucormycosis in Iran: A six-year retrospective experience. J Mycol Med. 2018;28(2):269-73.

17. Kennedy KJ, Daveson K, Slavin MA, Hal SJ, Sorrell TC, Lee A, et al. Mucormycosis in Australia: contemporary epidemiology and outcomes. Clin Microbiol Infect. 2016;22(9):775-81.

18. Komur S, Inal AS, Kurtaran B, Ulu A, Uguz A, Aksu HS, Taşova Y. Mucormycosis: a 10-year experience at a tertiary care center in Turkey. Turk J Med Sci. 2016;46(1):58-62.
19. Zilberberg MD, Shorr AF, Huang H, Chaudhari P, Paly VF, Menzin J. Hospital days, hospitalization costs, and inpatient mortality among patients with mucormycosis: a retrospective analysis of US hospital discharge data. BMC Infect Dis. 2014;14:310.

20. Zaki SM, Elkholy IM, Elkady NA, Abdel GK. Mucormycosis in Cairo, Egypt: review of 10 reported cases. Med Mycol. 2014;52(1):73-80.

21. Petrikkos G, Skiada A, Sambatakou H, Toskas A, Vaiopoulos G, Giannopoulou M, et al. Mucormycosis: ten-year experience at a tertiary-care center in Greece. Eur J Clin Microbiol Infect Dis. 2003;22(12):753-6.

22. Pagano L, Valentini CG, Posteraro B, Girmenia C, Ossi C, Pan A, et al. Zygomycosis in Italy: a survey of FIMUA-ECMM. J Chemother. 2009;21(3):322-9.

23. Lanternier F, Dannaoui E, Morizot G, Elie C, Garcia HD, Huerre M, Bitar D, et al. A global analysis of mucormycosis in France: the RetroZygo Study (2005-2007). Clin Infect Dis. 2012;54(1):35-43.

24. Chakrabarti A, Singh R. Mucormycosis in India: unique features. Mycoses. 2014;57(3):85-90.

25. Ferguson BJ. Mucormycosis of the nose and paranasal sinuses. Otolaryngol Clin North Am. 2000;33(2):349-65.

26. Greenberg RN, Scott LJ, Vaughn HH, Ribes JA. Zygomycosis (mucormycosis): emerging clinical importance and new treatments. Curr Opin Infect Dis. 2004;17(6):517-25.

27. Roden MM, Zaoutis TE, Buchanan WL, Knudsen T, Sakisova TA, Schaufele RL, et al. Epidemiology and outcome of zygomycosis: a review of 929 reported cases. Clin Infect Dis. 2005;41:634-653.

28. Connor BA, Anderson RJ, Smith JW. Mucor mediastinitis. Chest. 1979;75(4):525-6.

29. Helenglass G, Elliott JA, Lucie NP. An unusual presentation of opportunistic mucormycosis. Br Med J. 1981;282(6258):108-9.

30. Tedder M, Spratt JA, Anstadt MP, Hegde SS, Tedder SD, Lowe JE. Pulmonary mucormycosis: results of medical and surgical therapy. Ann Thorac Surg. 1994;57(4):1044-50.

31. Latif S, Saffarian N, Bellovich K, Provenzano R. Pulmonary mucormycosis in diabetic renal allograft recipients. Am J Kidney Dis. 1997;29(3):461-4.

32. Lee FY, Mossad SB, Adal KA. Pulmonary mucormycosis: the last 30 years. Arch Intern Med. 1999;159(12):1301-9.

33. Davoudi S, Graviss LS, Kontoyiannis DP. Healthcare-associated outbreaks due to Mucorales and other uncommon fungi. Eur J Clin Invest. 2015 Jul;45(7):767-73.

34. Andresen D, Donaldson A, Choo L, Knox A, Klaassen M, Ursic C, et al. Multifocal cutaneous mucormycosis complicating polymicrobial wound infections in a tsunami survivor from Sri Lanka. Lancet. 2005;365(9462):876-8.

35. Warkentien T, Rodriguez C, Lloyd B, Wells J, Weintrob A, Dunne JR, et al. Invasive mold 
infections following combat-related injuries. Clin Infect Dis. 2012;55(11):1441-9.

36. Neblett FR, Benedict K, Bos J, Bennett SD, Lo YC, Adebanjo T, Etienne K, et al. Necrotizing cutaneous mucormycosis after a tornado in Joplin, Missouri, in 2011. N Engl J Med. 2012;367(23):2214-25.

37. Li DM, Lun LD. Mucor irregularis infection and lethal midline granuloma: a case report and review of published literature. Mycopathologia. 2012;174(5):429-39.

38. Bist SS, Varshney S, Bisht M, Gupta N, Bhatia R. Isolated palate ulcer due to mucormycosis. Indian $\mathrm{J}$ Otolaryngol Head Neck Surg. 2008;60(1):79-82.

39. Meis JF, Chakrabarti A. Changing epidemiology of an emerging infection: zygomycosis. Clin Microbiol Infect. 2009;15(5):10-4.

40. Sanofi National study. Screening India's twin epidemic (SITE). Sanofi. 2011;1-5.

41. Chakrabarti A, Dhaliwal M. Epidemiology of mucormycosis in India. Current Fungal Infect Rep. 2013;7:287-92.

42. Kung VL, Chernock RD, Burnham CD. Diagnostic accuracy of fungal identification in histopathology and cytopathology specimens. Eur J Clin Microbiol Infect Dis. 2018;37(1):157-65.

43. Frater JL, Hall GS, Procop GW. Histologic features of zygomycosis: emphasis on perineural invasion and fungal morphology. Arch Pathol Lab Med. 2001;125(3):375-8.

44. Hoeppli RJC. Histological observations in experimental Schistosomiasis Japonica. Chin Med J. 1932;46:1179-86.

45. Splendore A. Sobre a cultura d'una nova especie de cogumello pathogenico. Revista de Sociedade Scientifica de Sao Paulo. 1908;62:62-3.

46. Cornely OA, Alastruey IA, Arenz D, Chen SCA, Dannaoui E, Hochhegger B, et al. Global guideline for the diagnosis and management of mucormycosis: an initiative of the European Confederation of Medical Mycology in cooperation with the Mycoses Study Group Education and Research Consortium. Lancet Infect Dis. 2019;19(12):405-21.

47. Therakathu J, Prabhu S, Irodi A, Sudhakar SV, Yadav VK, Rupa V. Imaging features of rhinocerebral mucormycosis: A study of 43 patients.
The Egyptian J Radiol Nuclear Med. 2018;49 (2):447-52.

48. Mohanty A, Rohilla R, Meena S, Bora M, Singh A, Kaistha N, et al. Epidemiology of Mycotic Infections: Experience From A Tertiary Care Center Of Uttarakhand, India. Trop J Pathol Microbiol. 2021;7(3):93-8

49. Yang M, Lee JH, Kim YK, Ki CS, Huh HJ, Lee NY. Identification of mucorales from clinical specimens: a 4-year experience in a single institution. Ann Lab Med. 2016;36(1):60-3.

50. Lanternier F, Poiree S, Elie C, Garcia HD, Bakouboula P, Sitbon K, et al. Prospective pilot study of high-dose (10 mg/kg/day) liposomal amphotericin B (L-AMB) for the initial treatment of mucormycosis. J Antimicrob Chemother. 2015;70(11):3116-23.

51. Pagano L, Offidani M, Fianchi L, Nosari A, Candoni A, Picardi M, et al. Infection Program. Mucormycosis in hematologic patients. Haematologica. 2004;89(2):207-14.

52. Nosari A, Oreste P, Montillo M, Carrafiello G, Draisci M, Muti G, et al. Mucormycosis in hematologic malignancies: an emerging fungal infection. Haematologica. 2000;85(10):1068-71.

53. Ibrahim AS, Gebremariam T, Husseiny MI, Stevens DA, Fu Y, Edwards JE, et al. Comparison of lipid amphotericin B preparations in treating murine zygomycosis. Antimicrob Agents Chemother. 2008;52(4):1573-6.

54. Larkin JA, Montero JA. Efficacy and safety of amphotericin B lipid complex for zygomycosis. Infect Med. 2003;20:201-6.

55. Singh A, Mohanty A, Jha S, Gupta P, Kaistha N. Concomitant Mucormycosis with Aspergillosis in Patients with Uncontrolled Diabetes Mellitus: A Case Series. J Clinical and Diagnostic Res. 2021;15(2):1-3

Cite this article as: Mohanty A, Gupta P, Varshney S, Kabi A, Angral S. Breaking the mold: a brief review on the diagnostic and treatment approaches of mucormycosis. Int J Otorhinolaryngol Head Neck Surg 2021;7:1207-15. 